\title{
Isolation and identification of Clostridium spp. from infections associated with the injection of drugs: experiences of a microbiological investigation team
}

\author{
J. S. BRAZIER, B. I. DUERDEN, V. HALL, J. E. SALMON, J. HOOD*, M. M. BRETT†, \\ J. MCLAUCHLIN† and R. C. GEORGE +
}

PHLS Anaerobe Reference Unit, University Hospital of Wales, Cardiff CF14 4XW, *Department of Microbiology, Glasgow Royal Infirmary, Glasgow G4 OSF, †Food Safety Microbiology Laboratory and $\$$ Respiratory and Systemic Infection Laboratory, Central Public Health Laboratory, 61 Colindale Avenue London NW9 5DF

\begin{abstract}
Pathogenic species of the genus Clostridium may contaminate the materials used in the injection of drugs and under the right conditions may cause serious or life-threatening disease. $C$. novyi type A was implicated in an outbreak of severe infection with high mortality in injecting drug users who injected heroin extravascularly. The isolation of such highly oxygen-sensitive clostridia from clinical material may require adherence to enhanced methods and, once isolated, commercially available anaerobe identification kits alone may not give an accurate identification. Additional phenotypic tests that are useful in recognising the main pathogenic species are described. Differentiation of $C$. novyi type A from $C$. botulinum type $C$ in reference laboratories was based on $16 S$ rDNA sequence data and specific neutralisation of cytopathic effects in tissue culture.
\end{abstract}

\section{Introduction}

Anaerobic spore-forming bacilli of the genus Clostridium are ubiquitous in the environment, existing in the form of exo-spores that can remain viable indefinitely. If illicit substances used by injecting drug users (IDU) become contaminated with these spores, their injection, particularly non-intravenous injection, may result in severe illness or death such as occurred in parts of the UK during 2000. Between April and August 2000, a total of 108 cases of IDU-associated infections was identified, 60 in Scotland (including 50 in Glasgow), 22 in Dublin and 26 in England, and there were 43 deaths $[1,2]$. Typically, the illness was characterised by the development of moderate to severe inflammation at or near an injection site several days after extravascular injection of heroin that had been dissolved in heated acid. In the most severe cases this was followed by hypotension, multi-organ failure and death, despite intensive treatment. The experiences gained by microbiologists involved in the investigations into this outbreak are reviewed here.

Received 8 July 2002; accepted 21 July 2002.

Corresponding author: Dr J. S. Brazier (e-mail: brazier@, cardiff.ac.uk).
Infections among recreational drug users account for $31-58 \%$ of their hospital admissions and $19 \%$ of their deaths associated with intravenous, intramuscular or subcutaneous injection [3-5]. Bacterial infections at the site of injection can be of a minor nature with local abscess formation; however, if injected material or paraphenalia are contaminated with certain clostridial spores, severe illness may result. Once introduced into a suitably oxygen-reduced environment within live human or animal tissue, these spores can germinate and, in the right conditions, certain pathogenic species may liberate powerful exotoxins that can have severe and life-threatening effects.

The genus Clostridium contains several species of known potential pathogenicity for man and animals. The pathogenic clostridia include the gas gangrene group - C. perfringens, C. septicum, C. sordellii, $C$. novyi and $C$. histolyticum - and the causal organisms of tetanus ( $C$. tetani), botulism ( $C$. botulinum), clostridial food poisoning $(C$. perfringens) and antibiotic-associated diarrhoea and colitis ( $C$. difficile). The contamination of wounds with clostridial species and the development of severe infection has hitherto been associated mostly with injuries sustained as a result of warfare. Gas gangrene (clostridial myonecrosis) was a 
major problem in the trench warfare during World War I, when deep artillery (shrapnel) wounds were commonly contaminated with soil and faeces, two prime sources of clostridial spores [6]. However, the practice of injecting substances into muscle tissue mimics the risk factors of infection associated with the trauma of warfare and, if the injectate contains clostridial spores, serious infections akin to those of wartime may result.

The practice of injecting morphine under the skin was described as far back as the 1830s [7], and the first reports of infections in people addicted to morphine injection were of tetanus in 1876 [8]. By the 1950s in New York in the USA, drug addiction accounted for the majority of cases of tetanus $[9,10]$. This was thought to have been associated with the 'cutting' of the street heroin with quinine that enhanced abscess formation, thus creating conditions suitable for the germination of $C$. tetani spores. Laboratory investigation of suspected clostridial disease often involves isolation of these organisms from clinical material such as tissues or exudates by anaerobic culture methods. However, the aero-tolerance of the pathogenic clostridial species varies considerably and attention to strict handling protocols is essential for the isolation of the more oxygen-sensitive species.

\section{Investigations for Clostridium species in human tissue and heroin}

In the early investigations into sudden deaths and serious illness during 2000 in IDUs who injected heroin intramuscularly or subcutaneously, there was clinical evidence of oedema, necrotising fasciitis or myonecrosis (without gas production) related to the injection site, coupled with rapid systemic deterioration and death [11]. At the outset it was suspected that spore-forming bacteria could be of potential importance because it was common practice to heat the heroin in citric acid or lemon juice before injection. This combination of low $\mathrm{pH}$ (estimated to be about $\mathrm{pH}$ $2.1)$ and heat $\left(70-80^{\circ} \mathrm{C}\right)$ would almost certainly destroy most vegetative bacterial cells but probably not damage bacterial spores.

Little is known about the microbial content of heroin, but Tuazon et al. [12] found that spore-forming aerobes (Bacillus spp.) and the anaerobe $C$. perfringens ranked as first and third, respectively, of all the organisms isolated from 'street' heroin and associated paraphenalia. To help determine the cause of these episodes of severe illness and deaths during 2000, tissue samples were examined by enhanced anaerobic methods and anaerobic isolates were identified by a combination of phenotypic and genotypic methods. In the investigation for the presence of clostridial spores in ante- or postmortem tissues from IDU patients who had fulfilled the case definition, the following protocol was drawn up to maximise their recovery.

\section{Protocol adopted to maximise the growth of} clostridia from spores in clinical tissues

- Perform all manipulations inside an anaerobic chamber $\left(\mathrm{N}_{2} 80 \%, \mathrm{H}_{2} \quad 10 \%, \mathrm{CO}_{2} \quad 10 \%\right)$.

- Ante- or post-mortem tissue for culture should be examined visually for any areas with evidence of necrosis and a portion of such material should be excised aseptically. This tissue should be divided in two and each half placed in a sterile glass or heat-stable plastic tube of suitable size. An equal volume of Fastidious Anaerobe Broth (Lab M) should be added to both containers and homogenised as much as possible by vortex mixing. Hard tissue may need grinding. One tube of the homogenised tissue/broth suspension should be removed to a heating block set at $80^{\circ} \mathrm{C}$ for $10 \mathrm{~min}$. After heating, the container should be returned to the anaerobic chamber, and both the heat-shocked and non-heat-shocked suspensions should be inoculated on to Fastidious Anaerobe Agar (FAA Lab M) supplemented with horse blood $6 \%$ and also into a cooked meat broth; both should be incubated anaerobically. Material should also be inoculated on to blood agar and incubated aerobically and in air with $\mathrm{CO}_{2} 5 \%$ in parallel. All plates and broths should be incubated at $37^{\circ} \mathrm{C}$. Any materials left should be mixed with an equal volume of a cryopreservative such as glycerol and frozen at $-80^{\circ} \mathrm{C}$.

- All anaerobic, aerobic and aerobic $+\mathrm{CO}_{2}$ cultures should be examined daily for up to 10 days. Any isolates on aerobic or aerobic $+\mathrm{CO}_{2}$ plates should be identified by standard laboratory protocols.

- If no anaerobic growth appears after that time, both heated and non-heated tissue in cooked meat broths should be subcultured to FAA blood agar and incubated as described above.

\section{Recognition of pathogenic clostridial species associated with wound infection}

\section{C. novyi type $A$}

C. novyi type A may be widely distributed in soil and, in one study, was present in $88 \%$ of samples examined [13]. When an anaerobic chamber is used for isolation, anaerobic cultures for $C$. novyi type A should be examined after overnight incubation within the anaerobic chamber for any small, flat, rough or rhizoidal, translucent, haemolytic colonies with a spreading edge. It is advised that plates should not be removed from the anaerobic chamber at this stage, as exposure to air would be toxic to any micro-colonies that have not begun sporulation. If anaerobic jars are used, these should be left unopened for at least $48 \mathrm{~h}$ before the plates are examined. After incubation for 48-72 h, colonies will often coalesce to give a fine spreading growth that may cover the entire plate, often with a marked $\beta$-haemolysis so as to make the blood agar plate completely transparent. Gram's staining shows 
gram-variable rods with occasional sub-terminal spores. A characteristic of $C$. novyi type A is the production of both lecithinase $\mathrm{C}$ and lipase on egg-yolk agar. The indole reaction can be variable and it can be difficult to differentiate $C$. novyi type A from $C$. botulinum type C. It may require specific neutralisation of the cytopathic effect of alpha toxin in bioassay, or $16 \mathrm{~S}$ rDNA sequence analysis to differentiate between these species. C. novyi type A is usually unreactive in commercial anaerobe identification kits and commonly is not identified by this approach. C. novyi type B has different phenotypic characteristics and can be distinguished by its biochemical reactions. The $C$. novyi type A isolates in the IDU outbreak were variable indole producers and could not be differentiated from $C$. botulinum type $\mathrm{C}$ by phenotypic tests alone. Definitive identification was obtained by $16 \mathrm{~S}$ rDNA gene sequence analysis [14] and detection of alpha toxin in bioassay. Cell-free culture supernates of $C$. novyi isolates from IDUs produced identical cytopathic effects to the type strain of C. novyi (NCTC 538) in Vero cells and these effects were completely neutralised by the international standard for $C$. novyi, i.e., Gas Gangrene ( $\mathrm{Cl}$. oedematiens) Antitoxin (3rd British Standard Established 1966) (64/24). 16S rDNA gene sequence analysis gave $100 \%$ homology with $C$. novyi type A accession no. X68188 (NCTC 538) listed in the EMBL database but only $98 \%$ homology with that listed for C. botulinum type C.

\section{C. perfringens}

Although $C$. perfringens was isolated from several cases of infection in IDUs, this organism was not believed to be of general significance in the outbreak investigation as this organism is frequently found as a post-mortem contaminant, being part of the normal gut flora. This fast-growing species will usually produce quite large discrete colonies after overnight incubation. They may be flat and rough-edged or smooth and domed, and either non-haemolytic or with a narrow zone of complete haemolysis inside a larger zone of partial haemolysis. This 'target' haemolysis is more pronounced on sheep blood agar than on horse blood agar. Gram's staining shows straight-sided gram-variable rods with no spores. The classical method of identifying $C$. perfringens is the Nagler plate which is a half anti-alpha toxin egg-yolk plate that is streaked at $90^{\circ}$ diametrically with the test organism. After incubation, the lecithinase $\mathrm{C}$ (alpha toxin) produces an opaque zone on the half plate without antitoxin that is inhibited on the half plate with the antitoxin. It has been difficult recently in the UK to obtain good quality anti-alpha toxin to $C$. perfringens. Poorer quality antisera may give a partial inhibition of lecithinase that is not species specific and may lead to misidentification of $C$. bifermentans as $C$. perfringens, for example. However, colony morphologies are distinct and Gram's stain will aid differentiation of these two species, as $C$. bifermentans is usually a prolific producer of sub-terminal spores whereas $C$. perfringens does not sporulate in vitro on normal agar media. Also, the result of the indole test differentiates between the two species (Table 1).

\section{C. septicum}

This organism is another rapidly growing Clostridium species that usually produces a thick swarming growth that is quite haemolytic. In culture, it has no characteristic odour, and Gram's staining reveals gram-variable rods with numerous sub-terminal spores. It should be recognised by use of commercially available identification kits. The most common source of $C$. septicum isolates seen in recent years has been from blood cultures from patients with malignancies of the colon or caecum for which this organism is a marker [15]. It can also be isolated from cases of spontaneous gas gangrene of the abdomen that are usually fatal.

\section{C. botulinum}

The proteolytic types $\mathrm{A}, \mathrm{B}$ and $\mathrm{F}$ initially produce discrete rhizoidal colonies that spread and coalesce. Haemolysis is variable, but the odour is strong and redolent of rotten eggs due to production of $\mathrm{H}_{2} \mathrm{~S}$. Gram's staining shows profuse sub-terminal and free spores and gram-variable bacilli. Phenotypically, they are identical to $C$. sporogenes and differentiation requires demonstration and specific neutralisation of botulinum toxin in a mouse assay. The non-proteolytic types of $C$. botulinum vary in their phenotypic characteristics but require similar toxin assays. In addition to food-borne illness, C. botulinum may be

Table 1. Simple tests to differentiate the pathogenic species of clostridia associated with wound infection

\begin{tabular}{lccccc}
\hline Species & Spores & Lecithinase & Lipase & Indole & Urease \\
\hline C. novyi A & ST & + & + & $\mathrm{V}$ & - \\
C. perfringens & - & + & - & - & - \\
C. septicum & $\mathrm{ST}$ & - & - & - & - \\
C. botulinum (types A, & $\mathrm{ST}$ & - & + & - & - \\
B. F proteolytic) & & - & - & $\mathrm{V}$ & - \\
C. tetani & $\mathrm{T}$ & - & - & + & $+/-$ \\
C. sordelliilbifermentans & $\mathrm{C} / \mathrm{ST}$ & + & - & \\
\hline
\end{tabular}

Spore position: ST, sub-terminal; T, terminal; C, central; V, variable. 
implicated in cases of wound botulism and such cases have occurred in IDUs [16].

\section{C. tetani}

No cases of tetanus associated with IDU have been reported recently, probably as a result of the success of the immunisation programme. Colonies of $C$. tetani may produce a fine swarming growth that can be easily missed on cursory examination. Gram's staining of overnight cultures often reveals readily overdecolorised long bacilli without spores. Further incubation yields the classical 'drum stick' appearance of cells with terminal, round spores. Phenotypically it is quite unreactive although some strains produce indole, and attempts at identification by commercial kits often require supplementation to confirm its identification. However, not all strains of $C$. tetani are toxigenic and referral for toxin bioassay may be required.

\section{C. sordellii}

This organism has been reported in other IDUassociated infections [17] and recently has been implicated in a fatal post-knee transplant infection the USA [18]. Colonies are large, grey-white and irregular, sometimes with a 'fern-leaf' edge. They produce indole and lecithinase but are urease negative, which differentiates them from $C$. bifermentans, generally regarded as a non-pathogen.

\section{Discussion}

The outbreak of a clostridial infection among IDUs in $2000[1,2]$ served to remind practising clinical microbiologists of some salient lessons. The fact that serious infections and deaths occurred as a result of injecting material containing clostridial spores into human tissue reminds us that so-called 'old' clostridial diseases are not consigned to history. New lifestyle practices can provide new opportunities for old diseases to re-emerge in a new setting. Hence, it is not just bullet, shrapnel and blast wounds that are of high risk of infection with clostridia. Although bacterial infections associated with drug injection are not a new phenomenon, the outbreak in 2000 was the first reported outbreak of infection amongst IDUs to involve C. novyi type A. Since the two World Wars, human infections with $C$. novyi type A have been reported rarely in the literature, and the PHLS Anaerobe Reference Unit for England and Wales has seen very few referrals of this organism in the last 20 years. Before the IDU outbreak, the last referral of C. novyi A type A was from a blood culture in a 23-year-old woman from Ireland in 1995, and before that from an infected crush injury to a thumb in an agricultural worker in 1990. Therefore, it is not surprising that diagnostic laboratories are unfamiliar with this organism and the fastidious conditions required for culture, especially on primary isolation. This outbreak also demonstrated the need for strict anaerobic techniques in order to isolate these very oxygen-sensitive bacteria. Laboratories used to isolating $C$. perfringens from clinical material may be lulled into thinking their anaerobic techniques are adequate, whereas this very aero-tolerant species is not a good marker for optimal anaerobic technique.

Even after clostridia had been isolated from IDU infections, most diagnostic laboratories encountered difficulties in attempting definitive identification to species level. Some commercially produced anaerobe identification kits could not recognise $C$. novyi type A. This over-reliance on kit-based identification demonstrates the move by clinical microbiology laboratories away from 'traditional' methods of bacterial identification based on in-house phenotypic tests coupled with the use of a good identification manual or textbook. In the case of $C$. novyi type $\mathrm{A}$, a strong clue to its recognition could be obtained by inoculation on to eggyolk agar. This organism is unusual in that it usually produces both lecithinase and lipase. The difficulties of identifying this organism were highlighted more generally when it was issued by the UK National External Quality Assessment Scheme (NEQAS) for microbiology on four occasions during the period 1989-1999. Over the four issues, an average of only $16 \%$ of reporting laboratories correctly identified $C$. novyi type $\mathrm{A}$, and an average of $15 \%$ reported the wrong species; $89 \%$ of laboratories did not go further than reporting 'Anaerobes isolated'.

In summary, colonies suspected of being clostridia isolated from wounds (especially those associated with drug abuse) usually require identification to species level to assess their potential significance. However, this often proves to be difficult for those clinical diagnostic laboratories that rarely encounter the pathogenic species. For confirmation of $C$. perfringens, many laboratories still rely solely on the Nagler reaction, sometimes with erroneous results. Although anaerobe identification kits may be of some assistance, they require careful interpretation and often require augmentation with further biochemical tests such as analysis of end-products of metabolism by gas-liquid chromatography and other simple tests as listed in Table 1. Molecular methods of identifying clostridia by $16 \mathrm{~S}$ rDNA sequence data analysis are feasible, but in practice not attainable by most clinical diagnostic microbiology laboratories. Therefore, any putative clostridia should be referred to a specialist laboratory for identification of anaerobic bacteria.

From the reference laboratory's point of view, this outbreak clearly demonstrated the value of full $16 \mathrm{~S}$ rDNA sequence analysis $(c .1400 \mathrm{bp})$ for definitive identification of some very closely related clostridial species. There is much phenotypic similarity between some of the species and they may differ from each 
other only by the production of certain large clostridial toxins that can be mediated by variable extrachromosomal genetic elements such as transposons, insertion sequences or bacteriophages. In addition to bioassay for specific toxin neutralisation tests, the $16 \mathrm{~S}$ rDNA database is an invaluable tool in distinguishing between these species and, in the outbreak investigation, the $16 \mathrm{~S}$ rDNA data completely matched the $C$. novyi alpha toxin neutralisation tests. Furthermore, the $16 \mathrm{~S}$ rDNA sequence data of the various $C$. novyi isolates from different towns and cities involved in this outbreak, hinted at differences that were largely borne out by the typing investigations of McLauchlin et al. [19].

Diagnostic microbiology laboratories should remain alert for clinical histories suggestive of injecting drug use and should employ optimal anaerobic methods to isolate any exacting clostridial species that may be present. They may also need to address the improvement of their clostridial identification skills to assess the potential significance of their findings.

We thank Christine Walton of NEQAS for the data on issues from the PHLS Quality Assurance Laboratory containing C. novyi.

\section{References}

1. McGuigan CC, Penrice GM, Gruer L, et al. Lethal outbreak of infection with Clostridium novyi type A and other sporeforming organisms in Scottish injecting drug users. $J$ Med Microbiol 2002; 51: 971-977.

2. Jones JA, Salmon JE, Djuretic T, Nichols G, George RC, Gill ON. An outbreak of serious illness and death among injecting drug users in England during 2000. J Med Microbiol 2002; 51: 978-984.
3. Scheidegger $\mathrm{C}$, Zimmerli W. Infectious complications in drug addicts: seven-year review of 269 hospitalized narcotic abusers in Switzerland. Rev Infect Dis 1989; 11: 486-493.

4. White AG. Medical disorders in drug addicts: 200 consecutive admissions. JAMA 1973; 223: 1469-1471.

5. Helpern M, Rho YM. Deaths from narcotism in New York City. Incidence circumstances and postmortem findings. NY State Med J 1966; 66: 2391-2408.

6. MacLennan JD. The histotoxic clostridial infections of man. Bacteriol Rev 1962; 26: 177-274.

7. Berridge V, Edwards G. Opium and the people - opiate use in nineteenth century England. London, Allen Lane. 1981.

8. Norman B. Tetanus after hypodermic injection of morphine. Lancet 1876; 4: 873.

9. Sapira JD. The narcotic addict as a medical patient. Am J Med 1968; 45: 555-588.

10. Siegel H, Helpern M, Ehrenreich T. The diagnosis of death from intravenous narcotism with emphasis on the pathologic aspects. J Forensic Sci 1966; 11: 1-16.

11. Anon. Serious unexplained illness among drug injectors. Commun Dis Rep CDR Wkly 2000; 10: 203, 206.

12. Tuazon CU, Hill R, Sheagren JN. Microbiologic study of street heroin and injection paraphenalia. J Infect Dis 1974; 129: $327-329$.

13. Nishida S, Nakagawara G. Isolation of toxigenic strains of Clostridium novyi from soil. J Bacteriol 1964; 88:1636-1640.

14. Hutson RA, Thompson DE, Collins MD. Genetic interrelationships of sacharolytic Clostridium botulinum types B, E and F and related clostridia as revealed by small-subunit rRNA sequences. FEMS Microbiol Lett 1993; 108: 103-110.

15. Koransky JR, Stargel MD, Dowell VR. Clostridium septicum bacteremia. Its clinical significance. Am J Med 1979; 66: 63-66.

16. Scheibe F, Hug B, Rossi M. [Wound botulism after drug injection.] Dtsch Med Wochenschr 2002; 127:199-202.

17. Bangsberg DR, Rosen JI, Aragon T, Campbell A, Weir L, Perdreau-Remington F. Clostridial myonecrosis cluster among injection drug users: a molecular epidemiology investigation. Arch Intern Med 2002; 162: 517-522.

18. Anon. Unexplained deaths following knee surgery - Minnesota, November 2001. Morb Mortal Wkly Rep 2001; 50: 1035-1036.

19. McLauchlin J, Salmon J, Ahmed S et al. Amplified fragment length polymorphism (AFLP) analysis of Clostridium novyi, Clostridium perfringens and Bacillus cereus isolated from injecting drug users during 2000. J Med Microbiol 2002; 51: 990-1000. 